higher percentage of atrial fibrillation and chronic obstructive pulmonary disease in AID patients. Compared to non-AID patients, AID patients had similar clinical ACS presentation and no differences were found with respect to revascularization strategies or medical treatment at discharge. With respect to prognosis the two groups had comparable rates of adverse events during hospitalization ( $10 \%$ vs $10 \%, p=0.920$ ) with no statistically significant differences in any single event studied. However after a follow-up of 397 [375-559] years, AID patients had higher rate of combined adverse events $(44 \%$ vs $28 \% p<0,001)$. After multivariate adjustment the presence of AID was associated with increased total mortality (hazard ratio $2.1,95 \% \mathrm{Cl} 1.2$ to $3.7, \mathrm{p}=0.008$ ) and it was also a borderline risk factor for higher bleeding complications (hazard ratio $2.2,95 \% \mathrm{Cl} 0.9$ to 5.5 ). The presence of AID was not an independent risk factor for neither stroke or recurrent non-fatal myocardial infarction.

Conclusions: The presence of AID did not change ACS presentation and clinical management. Although AID is not associated with worse outcomes during hospitalization it is independently linked to higher total mortality and a trend to an increased risk of major bleeding during follow-up.

Disclosure of Interest: None declared

DOI: 10.1136/annrheumdis-2017-eular.6758

\section{SAT0705 DO DIET CHARACTERISTICS IMPACT EFFECT OF A 12-MONTH VITAMIN D SUPPLEMENTATION ON 25OH-VITAMIN D SERUM LEVEL AMONG OVERWEIGHT ELDERLY?}

R.M. Baddoura ${ }^{1}$, N.A. Yaghi ${ }^{2}$, A. Arabi ${ }^{3}$, G. Halaby ${ }^{4}$, G. El-Hajj-Fuleihan ${ }^{3}$. ${ }^{1}$ Rheumatology, Hotel-Dieu de France, St Joseph University; ${ }^{2}$ Nutrition, School of Pharmacy, St Joseph University; ${ }^{3}$ Calcium Metabolism, American University of Beirut Medical Center; ${ }^{4}$ Endocrinology, Hotel-Dieu de France, St Joseph University, Beirut, Lebanon

Background: Achieving desirable 250 Hvitamin $D$ serum levels with vitamin D supplementation may be influenced by many confounders including lifestyle patterns and vitamin D receptor genetic polymorphisms.

Objectives: To test the effect of dietary intake confounding variables such total as calories, dietary intake of vitamin $\mathrm{D}$, calcium, proteins, carbohydrates and fats, on the variance in serum 25-hydroxy vitamin D (25OHD) level after vitamin D supplementation

Methods: Study population: All subjects from one out of three centers who participated in a randomized clinical trial comparing high (3750 IU/day) versus low (600 IU/day) dose of vitamin D supplementation in ambulatory overweight (BMI exceeding 25) elderly subjects (age $\geq 65$ years), with serum 25OHD level between 10 and $30 \mathrm{ng} / \mathrm{ml}$ at screening.

Outcome measure: $25 \mathrm{OH}$ D serum levels at 12 months of follow-up.

Confounders included gender, body mass index, baseline 25OHD serum level, genetic polymorphism of vitamin D receptor (Fok, Bsml and Taq) and dietary intake of vitamin D, calcium, proteins, carbohydrates, fats and total calories assessed by food frequency and $24 \mathrm{~h}$ recall questionnaires, administered a year after the termination of the trial.

Statistical analysis: ANOVA for repeated measures and regression using STATA version 13. Numbers expressed as mean (SD).

Results: We studied 117 Participants, 62 females and 55 males, age was 71 (5) years, of who 61 received 3,750 IU of vitamin D, and 55 received 600 IU/day. Vitamin D receptor genetic polymorphisms distribution was comparable in both arms. Daily vitamin D intake excluding provided supplements was 7.59 (1.21) $\mathrm{mcg}$ and $8.53(1.36) \mathrm{mcg}$ from the $24 \mathrm{~h}$ recall, and food frequency questionnaires, respectively ( $p$ was NS between the two arms). Calories, calcium, protein, carbohydrate and fat mean intake was also similar across both arms. The high-dose group achieved significantly higher $25 \mathrm{OH}$-vitamin $\mathrm{D}$ serum levels at twelve months $34.9(9.3) \mathrm{ng} / \mathrm{ml}$ compared to low-dose, $21.9(6.1) \mathrm{ng} / \mathrm{ml}$ $(p<0.0001)$. Difference between arms remained significant adjusting for gender, baseline BMI, Fok, Bsml and Taq genetic polymorphism, baseline serum vitamin $\mathrm{D}$ and dietary intake of vitamin D, calcium, calories, proteins, carbohydrates and fats. None of these dietary intake measures was found to have significant contribution to the model variance. Supplementation dose was the only significant predictor of 250 Hvitamin D serum levels achieved at 12 months.

Conclusions: Diet composition was not a confounder for the effect of high versus low dose vitamin D 12-month supplementation, on achieved 25OHD vitamin D serum levels.

Acknowledgements: St Joseph University, Hotel-Dieu de France hospital

American University of Beirut Medical Centre

Lebanese National Council for Scientific Research

Lebanese Ministry of Social Affairs

Disclosure of Interest: None declared

DOI: 10.1136/annrheumdis-2017-eular.2206

\section{SAT0706 END-STAGE ORGAN FAILURE IN SARCOIDOSIS: CHARACTERIZATION AND PREDICTIVE FACTORS IN 1082 PATIENTS}

R. Pérez-Alvarez ${ }^{1}$, B. Kostov ${ }^{2}$, A. González García ${ }^{3}$, R. Gómez De La Torre ${ }^{4}$, M. Lopez Dupla ${ }^{5}$, B. De Escalante ${ }^{6}$, A. Alguacil ${ }^{7}$, J. Chara ${ }^{8}$, J. Velilla ${ }^{9}$, J. Rascón ${ }^{10}$, J.S. Garcia Morillo ${ }^{11}$, C. Feijoo ${ }^{12}$, E. Fonseca ${ }^{13}$, M. Bonet ${ }^{14}$,
J.L. Callejas ${ }^{15}$, G. de la Red ${ }^{16}$, A.B. Madroñero Vuelta ${ }^{17}$, C. Soler ${ }^{18}$, E. Peral ${ }^{19}$ J.F. Gómez Cerezo ${ }^{20}$, G. Cruz ${ }^{21}$, P. Perez ${ }^{22}$, S. Rodríguez Fernández ${ }^{23}$, B. Pinilla ${ }^{24}$, A. Gato ${ }^{25}$, M. Akasbi ${ }^{26}$, A. Robles ${ }^{27}$, I. Ojeda ${ }^{28}$, M.J. Vives ${ }^{29}$, C. Morcillo ${ }^{30}$, M. Penadés ${ }^{31}$ M. De Vicente ${ }^{32}$, M. Ramos-Casals ${ }^{33}$,

L. Pallarés ${ }^{10}$, P. Brito-Zerón ${ }^{30,33}$ on behalf of the SARCOGEAS-SEMI Registry. ${ }^{1}$ Hosp Alvaro Cunqueiro, Vigo; ${ }^{2}$ IDIBAPS, Barcelona; ${ }^{3}$ Hosp Ramón y Cajal, Madrid; ${ }^{4}$ HUCA, Oviedo; ${ }^{5}$ Hosp Joan XXIII, Tarragona; ${ }^{6}$ Hosp Clínico, Zaragoza; ${ }^{7}$ Hosp Virgen de la Salud, Toledo; ${ }^{8}$ Hosp Josep Trueta, Girona; ${ }^{9}$ Hosp Miguel Servet, Zaragoza; ${ }^{10}$ Hosp Son Espases, Palma de Mallorca; ${ }^{11}$ Hosp Virgen del Rocio, Sevilla; ${ }^{12}$ Hosp Parc Taulí, Sabadell; ${ }^{13}$ Hosp de Cabueñes, Gijón;

${ }^{14}$ Althaia, Manresa; ${ }^{15}$ Hosp Clínico San Cecilio, Granada; ${ }^{16}$ Hosp Esperit Sant, Santa Coloma; ${ }^{17}$ Hosp San Jorge, Huesca; ${ }^{18}$ Hosp de Sta Caterina, Girona:

${ }^{19}$ Hosp Virgen Macarena, Sevilla; ${ }^{20}$ Hosp Infanta Sofía, Madrid; ${ }^{21}$ Hosp de Poniente, Almería; ${ }^{22}$ Hosp Puerta del Mar, Cádiz; ${ }^{23}$ Hosp da Barbanza, A Coruña; ${ }^{24}$ Hosp Gregorio Marañón, Madrid; ${ }^{25} \mathrm{CH}$, Albacete; ${ }^{26}$ Hosp Infanta Leonor; ${ }^{27}$ Hosp la Paz, Madrid; ${ }^{28}$ Hosp Valle del Guadiato, Córdoba; ${ }^{29}$ San Joan de Déu, San Boi; ${ }^{30}$ Hosp CIMA-Sanitas, Barcelona; ${ }^{31}$ Hosp de Manises, Valencia; ${ }^{32}$ Hosp Nuestra Señora del Prado, Talavera; ${ }^{33}$ Hosp Clínic, Barcelona, Spain

Objectives: To characterize the main features at presentation of sarcoidosis associated with the development of end-stage organ failure in a large multicenter cohort of patients from Southern Europe.

Methods: In January 2017, the Spanish National Registry of Sarcoidosis (SARCOGEAS-SEMI) included 1082 consecutive patients diagnosed with sarcoidosis according to the ATS/ERS/WASOG 1999 statement and extrathoracic involvement to the 2014 WASOG instrument. The development of end-stage organ failure was assessed at the last visit.

Results: The cohort consisted of $618(57 \%)$ women and $464(43 \%)$ men, with a mean age at diagnosis of $47 y$ rs. After a mean follow-up of 82 months, 90 (8\%) patients developed end-stage organ failure, including respiratory failure $(n=56)$, chronic renal failure $(n=13)$, cardiac failure/permanent cardiac device $(n=8)$ and liver cirrhosis $(n=3)$. The following baseline features were associated with end-stage organ failure in the univariate analysis: patients born in Spain $(p=0.008)$, a higher mean age at diagnosis $(p<0.001)$ and a radiological stage III/IV $(p<0.001)$ With respect to extrathoracic involvement, spleen $(p=0.015)$, renal $(p=0.001)$, cardiac $(p=0.028)$ and bone marrow $(p=0.003)$ involvements, hypercalcemia $(p=0.018)$ and use of corticosteroids $(p<0.001)$ were associated with end-stage organ failure, while patients with cutaneous sarcoidosis had a lower risk $(\mathrm{p}=0.029)$. Multivariate analysis identified age at diagnosis (OR 1.05), radiological stages III/IV (OR 3.12) and use of corticosteroids (OR 4.55) as independent variables associated with the development of end-stage organ failure.

Conclusions: Nearly $10 \%$ of patients with sarcoidosis developed end-stage organ failure. Respiratory failure represented two thirds of cases of sarcoidosis-related organ failure, followed by renal ( $15 \%$ of cases) and cardiac $(9 \%)$. Older patients, as well as those presenting with advanced radiological stages, had an enhanced risk of developing end-stage organ failure.

Disclosure of Interest: None declared

DOI: 10.1136/annrheumdis-2017-eular.3376

\section{SAT0707 EFFICACY AND SAFETY OF PROPHYLACTIC TREATMENT ON ACTIVIATION OF LATENT TUBERCULOSIS DURING GLUCOCORTICOID THERAPY IN PATIENTS WITH RHEUMATIC DISEASES}

R. Wu. Department of Rheumatology, the First Affiliated Hospital of Nanchang University, Nanchang, China

Background: More than $80 \%$ of cases of tuberculosis are the result of reactivated latent infection, and nearly all these cases could be prevented by the administration of a course of antibiotic treatment. Therefore, it is suggested screening and treatment are most beneficial for those patients taking immunosuppressive medications. Although the prevalence of latent tuberculosis infection in China was much higher than the United States on the basis of tuberculin skin testing, In China, so far, no study has been done about prophylactic treatment on prevention of tuberculosis activation during glucocorticoid therapy in patients with rheumatic diseases. Should it been recommended that all patients with rheumatic diseases be screened for the presence of active tuberculosis or LTBI before the use of long term glucocorticoid or immunosuppressive medications in China? It is reported only about $5 \%$ of immunocompetent persons with a positive test will have progression from latent infection to disease in their lifetime. and decisions about whether to treat latent tuberculosis should take into account the individual patient's risk for the development of active tuberculosis and the risks of therapy. Objectives: To evaluate the risk of reactivation of LTBI in patients with rheumatic diseases who were undergoing prednisone use and the efficacy and safety of prophylactic treatment on prevention of tuberculosis reactivation.

Methods: 1000 patients with rheumatic diseases who were treated with prednisone were enrolled since 2012. IGRA test (the T-SPOT.TB test) were performed for all subjects. 50 patients with a IGRA-positive were administrated with rifampin for 4 months. 2-years follow-up was conducted to evaluate the risk factors of reactivation of LTBI and the efficacy and safety of rifampin treatment on prevention of tuberculosis reactivation. 
Results: the IGRA-positive rates were $20.6 \%$ (206/1000). Forty-eight patients with a IGRA-positive finished the treatment with rifampin for 4 months. 2-years follow-up shows no latent tuberculosis developed into active tuberculosis in the patients with prevention of rifampin, while $3(0.38 \%)$ patients with negative IGRA and $22(10.7 \%)$ patients with positive IGRA without rifampin treatment developed into active tuberculosis. 25 patients had active tuberculosis disease in two years, which 24 patients with SLE and one patient with systemic vasculitis while 20 cases had pulmonary tuberculosis, 3 cases had vertebral tuberculosis and tuberculous peritonitis, tuberculous meningitis was 1 case, respectively. Univariate analysis showed that age, entities of rheumatic disease, dosage of glucocorticoid, DMARDs using, comorbidity with interstitial lung disease and cancer were significantly associated with tuberculosis activation $(P<0.05)$, with the ORs of $0.959,0.592,4.45,0.226, A 3.51$ and 69.9 , respectively. Entities of rheumatic diseases, Dosage of glucocorticoid, DMARDs using, comorbidity of cancer entered the final multivariate Logistic model. No severe adverse effects occurred in all subjects.

Conclusions: Medium or high dosage of glucocorticoid treatment appears to increase the risk of activation of latent tuberculosis infection. Latent tuberculosis activation could be safely prevented by 4-months rifampin treatment while starting glucocorticoid and DMARDs therapy.

Disclosure of Interest: None declared

DOI: 10.1136/annrheumdis-2017-eular.3937

\section{SAT0708 SYMPTOMS INDICATIVE OF INFLAMMATORY ARTHRITIS ARE COMMON IN THE PRIMARY CARE POPULATION: FINDINGS FROM THE SYMPTOMS IN PERSONS AT RISK OF RHEUMATOID ARTHRITIS SURVEY}

S.L. Hider ${ }^{1,2}$, S. Muller ${ }^{1}$, J. Prior ${ }^{1}$, T. Helliwell ${ }^{1}$, D. van Schaardenburg ${ }^{3}$, A. van der Helm-van Mil ${ }^{4}$, K. Raza ${ }^{5}$, C.D. Mallen ${ }^{1} .{ }^{1}$ Arthritis Research UK Primary Care Centre, Keele University, Keele; ${ }^{2}$ Haywood Academic Rheumatology Centre, Haywood Hospital, Staffordshire, United Kingdom; ${ }^{3}$ Reade, Amsterdam; ${ }^{4}$ Department Rheumatology, Leiden University Medical Centre, Leiden, Netherlands: ${ }^{5}$ Rheumatology Research Group, College of Medical and Dental Sciences, University of Birmingham, Birmingham, United Kingdom

Background: Early, accurate diagnosis of RA is critical to improving outcomes. Patients with RA may develop a variety of symptoms including joint pain, swelling and stiffness. The Symptoms in Persons at Risk of Rheumatoid Arthritis (SPARRA) questionnaire was derived to assess the presence, severity and impact of common symptoms in patients at risk of RA (1). However, to date there is little data available on how common these symptoms are in primary care consulters. Objectives: To describe the prevalence of self-reported inflammatory joint symptoms in primary care patients consulting for both musculoskeletal and non-musculoskeletal complaints.

Methods: Questionnaires were sent to 10161 individuals, of whom 5050 had consulted primary care for musculoskeletal problems. The remainder were matched to this sample by age, gender and general practice and had consulted for any non-musculoskeletal indication. Respondents provided data on presence of common symptoms such as joint pain, stiffness and swelling. The prevalence of these symptoms, their severity and impact was compared between musculoskeletal and non-musculoskeletal consulters.

Results: 4549 people responded to the survey (adjusted response $45.8 \%$ ) of whom $52.3 \%$ were in the musculoskeletal consultation group. The mean (SD) age was $61.6(14.8)$ years and $58.9 \%$ were female. Symptoms commonly associated with inflammatory arthritis were common in both groups. $89.1 \%$ of musculoskeletal consulters reported current joint pain, compared with $74.9 \%$ in the non-MSK consulter group, $48.7 \%$ of MSK consulters reported joint swelling compared to $37.3 \%$ of non-MSK consulters and $77.9 \%$ of MSK consulters reported joint stiffness $(64.3 \%$ in non-MSK group). A similar proportion in each group reported fatigue $(59.5 \%$ vs $55 \%)$. Joint symptoms remained common symptoms in both groups even when severity and impact of symptoms was considered.

Conclusions: Although symptoms such as joint pain, swelling, and stiffness are predictive of inflammatory arthritis, a large proportion of those consulting primary care for non-musculoskeletal reasons routinely report these symptoms when prompted. This compounds the challenges of diagnosing inflammatory arthritis in a non-specialist setting where new approaches are needed to ensure accurate, early diagnosis, facilitating a treat to target approach.

References:

[1] van Tuyl, et al. Musculoskeletal Care 2016;14:169-73.

Acknowledgements: This study represents independent research funded by the National Institute for Health Research (NIHR). TH is funded by a NIHR Clinical Lectureship in General Practice. CDM is funded by the National Institute for Health Research (NIHR) Collaborations for Leadership in Applied Health Research and Care West Midlands, the NIHR School for Primary Care Research and a NIHR Research Professorship in General Practice (NIHR-RP-2014-04-026). The views expressed are those of the authors and not necessarily those of the NHS, NIHR or the Department of Health.

Disclosure of Interest: None declared

DOI: 10.1136/annrheumdis-2017-eular.5503

\section{SAT0709 MORTALITY PREDICTION IN MIXED CONNECTIVE TISSUE DISEASE}

S. Reiseter ${ }^{1}$, R. Gunnarsson ${ }^{2}$, T.M. Aaløkken ${ }^{3}$, M.B. Lund ${ }^{4}$, J. Corander ${ }^{5}$, Ø. Molberg ${ }^{6} .{ }^{1}$ Institute of Clinical Medicine, University of Oslo, Norway; ${ }^{2}$ Dept. of Rheumatology; ${ }^{3}$ Dept. of Radiology and Nuclear Medicine; ${ }^{4}$ Dept. of Respiratory Medicine, Oslo University Hospital; ${ }^{5}$ Institute of Basic Medical Sciences;

${ }^{6}$ Institute of Clinical Medicine, University of Oslo, Oslo, Norway

Background: Mixed Connective Tissue Disease (MCTD) is a chronic, immune-mediated disorder defined by the combined presence of serum antiribonucleoprotein (RNP) antibodies and selected clinical features of Systemic Sclerosis, Systemic Lupus Erythematosus, Rheumatoid Arthritis and Polymyositis. Several clinical manifestations and laboratory findings have been found to be associated to increased risk of mortality in univariable analyses (1).

Objectives: Here we present a mortality predicting model in a long-term observational unselected nationwide cohort aiming to enhance the knowledge of long-term prognosis in MCTD.

Methods: 135 patients were included from our nationwide MCTD cohort. Abnormal high resolution computed tomography (CT) findings of ground glass attenuation and reticular patterns were defined as Interstitial Lung Disease (ILD) and expressed as percentage of Total Lung Volume (TLV). Pulmonary function tests and laboratory tests were performed within 2 months of the HRCT examination. Pleuritis was defined as typical pleurisity for more than one day, pleural effusions or pleural rub present at or before baseline. Pericarditis was defined as typical pericardial pain for more than one day, pericardial effusion, pericardial rub or pericarditis by electrocardiography at or before baseline. Myositis was confirmed by muscle biopsy and/or electromyogram and CK elevation at or before baseline. Cox regression analyses were used to find the predictive factors of mortality. Variables at a significant level of $P<.25$ where considered a candidate in the prediction model by manual backward elimination procedure.

Results: 21 patients died after a mean (standard deviation) observation of 9 (2) years. The predictive model is shown in Table 1. According to the Harrell's C index, patient outcomes were accurately predicted by this model $85 \%$ of the time.

Table 1

\begin{tabular}{lcccccc}
\hline & \multicolumn{3}{c}{ Univariable } & & \multicolumn{3}{c}{ Multivariable } \\
\hline & $\mathrm{HR}$ & $95 \% \mathrm{Cl}$ & $\mathrm{P}$ value & $\mathrm{HR}$ & $95 \% \mathrm{Cl}$ & P value \\
Pericarditis ever & 4.0 & $1.6-9.7$ & 0.003 & 5.5 & $1.9-16.0$ & 0.002 \\
Male gender & 2.5 & $1.1-5.9$ & 0.038 & 5.8 & $1.9-17.9$ & 0.002 \\
\% ILD of TLV & 1.1 & $1.03-1.1$ & 0.001 & 1.1 & $1.0-1.1$ & 0.010 \\
DLCO <60\% & 3.1 & $1.3-7.5$ & 0.011 & 3.1 & $1.0-9.2$ & 0.046 \\
Agegroups* at diagnosis & 1.8 & $1.4-2.4$ & $<0.001$ & 1.9 & $1.4-2.5$ & $<0.0001$ \\
Baseline ESR $>30 \mathrm{~mm}$ & 3.3 & $1.4-7.7$ & 0.007 & & & \\
FVC $<75 \%$ & 3.6 & $1.4-8.8$ & 0.006 & & & \\
Arthritis present at or before baseline & 2.1 & $0.85-5.2$ & 0.109 & & & \\
Pleuritis ever & 2.2 & $0.81-6.1$ & 0.121 & & & \\
Baseline Hb $<12 \mathrm{~g} / \mathrm{dL}$ & 2.7 & $1.0-7.0$ & 0.041 & & & \\
Myositis & 0.22 & $0.03-1.7$ & 0.144 & & &
\end{tabular}

*Patients were divided in 6 age groups at diagnosis ( $<25$ years, 26 to 35 years, 36 to 45 years, 46 to 55 years, $56-65$ years and above 65 years). FVC = Forced Vital Capacity \% of predicted, DLCO = diffusing capacity of the lung for carbon monoxide \% of predicted, ESR = erythrocyte sedimentation rate, $\mathrm{TLV}=$ total lung volume, $\mathrm{Hb}=$ Haemoglobin

Conclusions: The strongest predicting factors of mortality in MCTD is increasing $\%$ ILD of TLV, pericarditis, male gender, DLCO less than $60 \%$ of predicted and increasing age at diagnosis.

References:

[1] Hajas A, Szodoray P, Nakken B, Gaal J, Zold E, Laczik R, et al. Clinical course, prognosis, and causes of death in mixed connective tissue disease. The Journal of rheumatology. 2013:40(7):1134-42.

Disclosure of Interest: None declared

DOI: 10.1136/annrheumdis-2017-eular.1855

\section{SAT0710 RENAL FUNCTION CONTRIBUTE TO RISK OF CARDIOVASCULAR DISEASE IN RHEUMATOID ARTHRITIS}

S. Hannawi ${ }^{1}$, I. Alsalmi ${ }^{2}$. ${ }^{1}$ Rheumatology, Ministry of Health and Prevention of UAE, Dubai, United Arab Emirates; ${ }^{2}$ Renal, Royal Hospital, Muscat, Oman

Background: The excess mortality associated with rheumatoid arthritis (RA) is due largely to cardiovascular disease. This highest risk is not related primarily to traditional cardiovasculr/atherosclerosis risk factors. The presence of RA indepedently, as well as high inflammation associated with RA has been reported as a cardiovascular risk factors. Also, subclinical decreased kidney function has been identified as an independent risk factor for $\mathrm{CV}$ events in patients with RA. The potential impact of impaired kidney function on atherosclerosis in RA requires more elucidation.

Objectives: To assess the role of renal parameters, alongside with inflammation and traditional cardiovascular risk factors in predicting cardiovascular disease; as manifested by carotid intima media thickness (cIMT), among RA population.

Methods: cIMT measurement was carried out in 68 RA patients, and correlated with renal function parameters with adjustment for traditional CV risk factors and RA associated inflammation. Glomerular filtration rate (GFR) was estimated with the abbreviated Modification of Diet in Renal Disease formula. Linear regression determined the association between renal parameters and the thickness of cIMT. 